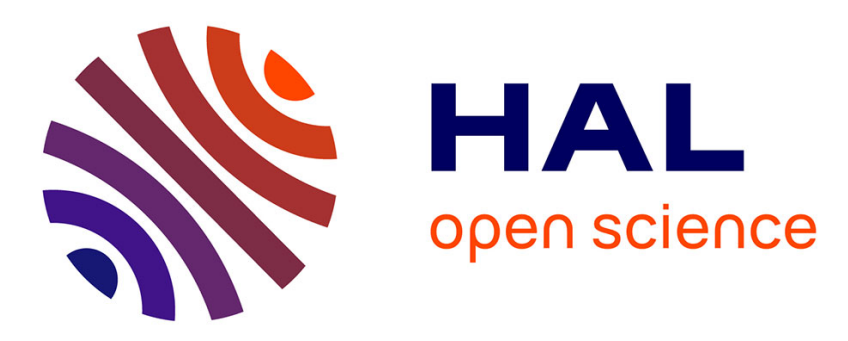

\title{
Limit design of axisymmetric shells with application to cellular cofferdams
}

\author{
P. de Buhan, Alain Corfdir
}

\section{To cite this version:}

P. de Buhan, Alain Corfdir. Limit design of axisymmetric shells with application to cellular cofferdams.

J. Engng. Mech. ASCE, 1996, pp.921-929. 10.1061/(ASCE)0733-9399(1996)122:10(921) . hal00111514

\section{HAL Id: hal-00111514 \\ https://hal.science/hal-00111514}

Submitted on 27 Aug 2019

HAL is a multi-disciplinary open access archive for the deposit and dissemination of scientific research documents, whether they are published or not. The documents may come from teaching and research institutions in France or abroad, or from public or private research centers.
L'archive ouverte pluridisciplinaire HAL, est destinée au dépôt et à la diffusion de documents scientifiques de niveau recherche, publiés ou non, émanant des établissements d'enseignement et de recherche français ou étrangers, des laboratoires publics ou privés. 


\title{
Limit Design of Axisymmetric Shells with Application to Cellular Cofferdams
}

\author{
By P. de Buhan ${ }^{1}$ and A. Corfdir ${ }^{2}$
}

\begin{abstract}
This paper is devoted to the limit design of cellular cofferdams that are regarded as mixed structures where the backfill is modeled as a three-dimensional continuum, while the surrounding sheet pile wall is treated as a cylindrical shell. Dealing with this structure from a static point of view, it turns out that the problem under consideration requires the calculation of the ultimate load value of a circular cylindrical shell subjected to a linearly varying pressure distribution representing the thrust of the backfill material. Extending the results of previous works, a complete solution to this problem is developed for different boundary conditions. The corresponding results are discussed, notably the influence of the shell relative thickness. They are applied to the design of a single cellular cofferdam whose stability under gravity forces is examined, with the strength of the granular backfill material being described by a Mohr-Coulomb criterion.
\end{abstract}

\section{INTRODUCTION}

Cellular cofferdams are civil engineering structures that are most commonly encountered in hydraulic works, where they provide a convenient means for isolating dry work sites in water-covered areas. They may also be used as breakwaters or retaining walls. They are constructed by assembling vertical metallic sheet piles, either driven into the soil or simply resting on it, in order to form circular cylindrical cells filled with a granular material. Although the intensive use of these kinds of structures is not new (it may even be traced back to the beginning of the century), their mechanical behavior is still to be fully understood, particularly their limit design.

Simplified engineering design rules are already available (see, for instance, Bowles 1977 or Lacroix et al. 1970), based on the idea that the thrust of the backfill material inside the cell generates interlocking tensions between the sheet piles, to which the latter should be able to resist. One of the difficult points is to decide which value of the backfill thrust should be taken into account in such an analysis. A first comprehensive approach that provides a rigorous mechanical framework to this problem has recently been developed by Dormieux and Delaurens (1991), whose analysis is based on the yield design (or limit design) theory (Salençon 1983, 1990, 1993). Making use of both the lower bound and upper bound methods, they derived crude but rigorous estimates for the stability factor of a single cell subjected to its own weight. In regard to the lower bound estimates that they obtained through the static approach, it should be noted that their calculation implicitly amounts to disregarding any resistance of the sheet piles to bending moments. It thus appears that a possible way to improve these estimates consists of taking the bending resistance into account. It is, therefore, necessary to get a better insight into the mechanical behavior of the sheet pile cell, which from now on will be modeled as a homogeneous cylindrical shell.

Limit analysis methods applied to plates and shells, regarded as two-dimensional continuous media, have formed the subject of many prominent works, among which one should

\footnotetext{
${ }^{1}$ Prof. in Struct. Des., École Nationale des Ponts et Chaussées, Head of CERCSO (Res. Ctr. for Struct. Des.), Laboratoire de Mécanique des Solides (URA 317 du CNRS), École Nationale des Ponts et Chaussées, La Courtine, 93167, Noisy-le-Grand Cedex, France.

${ }^{2}$ Res. Engr., Laboratoire de Mécanique des Solides (URA 317 du CNRS), École Nationale des Ponts et Chaussées, La Courtine, 93167, Noisy-le-Grand Cedex, France.
}

quote those of Drucker (1954), Hodge (1954, 1963), Onat and Prager (1954), Eason and Shield (1955), and Drucker and Shield (1959). Drucker and Shield (1959), for instance, proposed a simplified yield strength criterion for shells, involving no interaction between normal forces and bending moments. This criterion may be regarded as an approximation to the true strength criterion for a shell made of a homogeneous Tresca material. Using such a criterion, Olszak and Sawczuk (1959) have derived solutions to the classical problem of a tank submitted to a hydrostatic distribution of pressure. These results have been reported, with slight modifications, in Save and Massonnet (1972). Unfortunately, they cannot be directly applied to cellular cofferdams, since the analysis performed by Olszak and Sawczuk (1959) in the relevant situation (tank with free upper edge) was restricted to "short" shells; that is, to shells whose length is small compared to their radius (given a value of the thickness).

The main purpose of the present paper is to derive exact solutions to the aforementioned problem, for short as well as long cylindrical shells. Three kinds of boundary conditions concerning the lower edge of the shell will be successively examined. They are representative of different situations one may come across in practice, depending on the construction conditions of the cellular cofferdams. These solutions are first obtained by means of the yield kinematic approach, making use of failure mechanisms involving hinge circles, then by exhibiting generalized stress distributions along the shell, in order to prove that the lower and upper bound estimates coincide. The results so obtained make it possible to quite significantly improve previous lower bound estimates for the stability factor of cellular cofferdams.

\section{STABILITY ANALYSIS OF CELLULAR COFFERDAM: STATEMENT OF PROBLEM}

Cellular cofferdams are civil engineering structures comprised of a cylindrical shell made of metallic sheet piles connected to each other along the vertical direction, filled with a cohesionless granular material (sand or gravel) [Fig. 1(a)]. It is convenient to analyze the stability of such a structure by resorting to a so-called "mixed modeling" approach where the surrounding sheet pile wall is schematized as a cylindrical shell, while the backfill material is considered as a three-dimensional continuous medium whose strength properties are defined through a Mohr-Coulomb condition that writes

$$
f(\boldsymbol{\sigma})=\left(\sigma_{1}-\sigma_{3}\right)+\left(\sigma_{1}+\sigma_{3}\right) \sin \varphi \leq 0
$$

where $\boldsymbol{\sigma}=$ stress tensor at any point of backfill material; $\sigma_{1}$ and $\sigma_{3}=$ major and minor principal stresses, respectively (tensile stresses are counted as being positive); and $\varphi=$ friction 


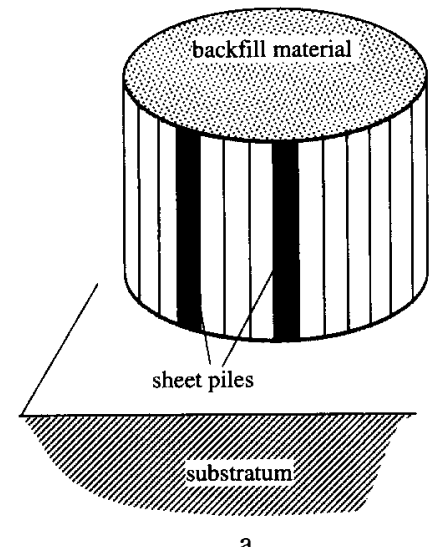

a

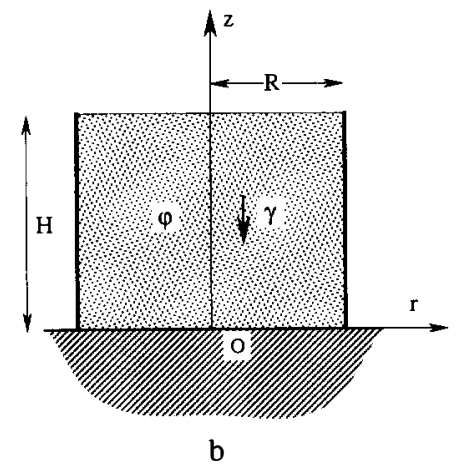

FIG. 1. Mixed Modeling of Cellular Cofferdam: (a) Basic Structure; (b) Coordinate System

angle. It is thus assumed that the backfill material exhibits no cohesion.

The whole structure is resting on a horizontal rigid bedrock. It is referred to as a cylindrical coordinate system $(r, \theta, z)$ with the origin being taken as the intersection of the lower plane with the symmetry axis. No surcharge is applied to the structure apart from the gravity characterized by a uniform field of body forces equal to $-\gamma e_{2}$, where $\gamma$ is the specific weight of the backfill material [Fig. 1(b)]. Concerning the sheet pile wall, modeled as a cylindrical shell of height $H$ and radius $R$, it will be assumed that no external load is applied on its upper edge $(z=H)$, while the following three kinds of boundary conditions will be considered in the sequel for the lower edge $(z=0)$ :

- Free edge - the lower end is free to move horizontally as well as to rotate

- Simply supported edge-the displacements are fixed equal to zero, while the rotation remains free

- Clamped edge - no translation and rotation are allowed

These boundary conditions may be considered as modeling, in a realistic way, the physical interaction between the sheet piles and the substratum. By way of illustration, the third boundary condition (clamped edge) corresponds, for instance, to the case when the sheet piles are driven deep enough into the substratum, while the free edge condition would be associated with negligible horizontal friction forces between the sheet pile extremities and the substratum. The second type of boundary condition represents an intermediate situation.

Taking into account the axisymmetry of the problem, it is advisable to consider distributions of stresses in the shell that also satisfy this symmetry.

In terms of normal and shear resultants as well as bending moments (referring to an infinitesimal shell element such as that shown in Fig. 2), the following components vanish:

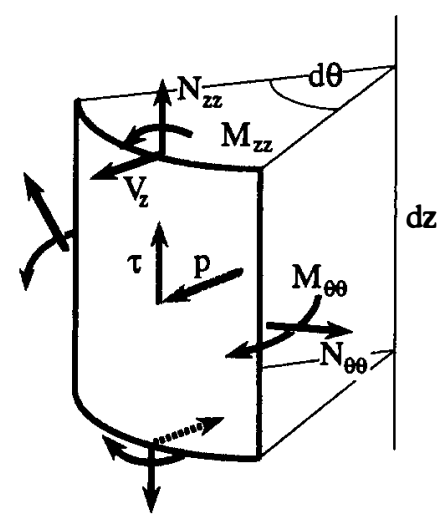

FIG. 2. Equilibrium of Infinitesimal Shell Element under Axisymmetric Conditions

$$
N_{\theta z}=N_{z \theta}=0 ; \quad M_{\theta z}=M_{z \theta}=0 ; \quad V_{\theta}=0
$$

Resorting to the same argument of axisymmetry, it appears that the stress vector $\mathbf{T}$ applied at any point of the shell by the backfill material is of the form

$$
\mathbf{T}=p(z) \mathbf{e}_{r}+\tau(z) \mathbf{e}_{z}
$$

Enforcing both the resultant and moment equilibrium of the shell element shown in Fig. 2 leads to the following equations:

$$
\begin{gathered}
\frac{d V_{z}}{d z}-\frac{N_{\theta \theta}}{R}+p(z)=0 ; \quad \frac{d M_{z z}}{d z}+V_{z}=0 \\
\frac{d N_{z z}}{d z}+\tau(z)=0 ; \quad \frac{d M_{\theta \theta}}{d \theta}=0
\end{gathered}
$$

Eliminating $V_{2}$ between $(4 a)$ and $(4 b)$, the following classical second-order differential equation is obtained:

$$
\frac{d^{2} M_{z z}}{d z^{2}}+\frac{N_{\theta \theta}}{R}-p(z)=0
$$

which may be found, for instance, in Chen and Han (1988).

Integrating (4c) over the height of the shell and taking into account the boundary conditions on the upper edge $\left[N_{z z}(z=\right.$ $H)=0$ ] yields

$$
N_{\mathrm{zz}}=-\int_{H}^{z} \tau\left(z^{\prime}\right) d z^{\prime}
$$

Eqs. (5) and (6) express the equilibrium of the shell subject to the stress distribution $p(z)$ and $\tau(z)$.

According to the yield design theory (or limit design) formulation (Salençon 1983, 1990, 1993), the stability of the whole structure is ensured if one can exhibit a stress distribution of membrane forces $\left(N_{z z}, N_{\theta \theta}\right)$ and moments $\left(M_{\theta \theta}, M_{z z}\right)$ in the shell, with both of them complying with equilibrium and strength requirements. Dealing with this problem by means of the static approach, a particular choice is now being made concerning the stress fields in the backfill material, defined as follows in the cylindrical coordinates:

$$
\begin{gathered}
\sigma_{z z}=\gamma(z-H), \sigma_{r r}=\sigma_{\theta \theta}=\lambda \sigma_{z z} \text { and } \\
\sigma_{i j}=0 \text { for the other components }
\end{gathered}
$$

where $\lambda$ represents an arbitrary constant.

These stress fields automatically satisfy the equilibrium equation (div $\left.\sigma-\gamma e_{z}=0\right)$ as well as the boundary conditions prescribed at $z=H$ (stress-free top level). Moreover, it complies with the strength condition (1) if and only if

$$
K_{a} \leq \lambda \leq K_{p}
$$

where $K_{a}=(1-\sin \varphi) /(1+\sin \varphi)$ and $K_{p}=K_{a}^{-1}$ denote 
classical active and passive earth pressure coefficients, respectively.

The corresponding stress distribution exerted on the surrounding shell is therefore

$$
\mathbf{T}=\boldsymbol{\sigma} \cdot\left(-\mathbf{e}_{r}\right)=-\lambda \gamma(z-H) \mathbf{e}_{r}
$$

That is, by comparing with (3)

$$
p=P\left(1-\frac{z}{H}\right) ; \quad \tau=0
$$

with $P=\lambda \gamma H$ representing maximum pressure applied to bottom of shell $(z=0)$.

It then remains to be seen whether the shell will be able to resist the linearly varying pressure distribution (10). This will now be considered in itself as an auxiliary yield design problem, where $P$ stands for loading parameter.

\section{YIELD DESIGN FORMULATION OF AUXILIARY PROBLEM}

The auxiliary problem under consideration is that of a cylindrical shell of height $H$ and radius $R$ subjected to an internal pressure distribution increasing linearly from zero at the top of the shell to a prescribed value denoted by $P$ at the bottom (Fig. 3). As a result of (6) with $z=0$, it appears that $N_{z z}=0$, so that the only stress components having nonzero values are $N_{\theta \theta}$ and $M_{z z}$ which fulfill equilibrium (5), while from (4d) $M_{\theta \theta}$ is an arbitrary function of $z$. Following Olszak and Sawczuk's (1959) approach, a simplified criterion limiting the values of $N_{\theta \theta}$ and $M_{z z}$, respectively, will be adopted

$$
\left|N_{\theta \theta}\right| \leq N_{0} ; \quad\left|M_{z z}\right| \leq M_{0}
$$

Such a criterion may be considered as an approximation of the actual strength criterion, which can be derived in the case of a shell made of a homogeneous Tresca material (Drucker and Shield 1959). More specifically, denoting by $\sigma_{0}$ the resistance of the constituent material under a uniaxial stress, it can be shown that (11) is an upper bound of the exact criterion if one takes $N_{0}=\sigma_{0} e$ and $M_{0}=\sigma_{0} e^{2} / 4$ ( $e$ is the thickness of the shell), while it turns out to be a lower-bound estimate when $N_{0}=(3 / 4) \sigma_{0} e$ and $M_{0}=(3 / 16) \sigma_{0} e^{2}$. In the case of a cellular cofferdam, the shell being constituted by an assemblage of metallic sheet piles, it seems reasonable, in the absence of more specific data, to adopt a simple criterion as (11), taking for $N_{0}$ the force per unit length along the connection between two sheet piles necessary for tearing them apart. This force can be measured through a very simple testing procedure.

Within the framework of the yield design theory, it appears that the ultimate load $P^{+}$is defined as the maximum value of $P$ for which one can exhibit at least one distribution of $N_{\theta \theta}$ and $M_{z z}$ statically admissible with $P$. Such a distribution is

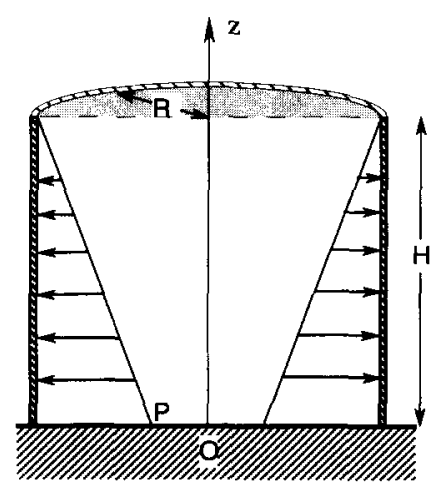

FIG. 3. Auxiliary Problem-Cylindrical Shell Submitted to Linear Distribution of Internal Pressure satisfying the equilibrium (5) together with the prescribed stress boundary conditions, while complying with the strength conditions (11) at every point of the structure. Introducing, for the sake of convenience, the following set of nondimensional variables

$$
u=\frac{z}{H} ; \quad n=\frac{N_{\theta \theta}}{N_{0}} ; \quad m=\frac{M_{z z}}{M_{0}} ; \quad k=\frac{M_{0} R}{N_{0} H^{2}} ; \quad \text { and } f=\frac{P R}{N_{0}}
$$

This problem reduces to searching for $f^{+}=P^{+} R / N_{0}$, defined as the maximum value of $f$ for which there exist two functions $n(u)$ and $m(u)$ defined on the interval $[0,1]$ such that

$$
\frac{d^{2} m}{d u^{2}}+\frac{n}{k}-\frac{f}{k}(1-u)=0 ; \quad|m| \leq 1 \text { and }|n| \leq 1
$$

and for the upper edge $(u=1)$

$$
m(1)=m^{\prime}(1)=0
$$

[Note that $m^{\prime}(1)=0$ is derived from condition $V_{z}(z=H)=0$ given in $(4 b)$, whereas for the lower edge $(u=0)$

$$
\begin{gathered}
\text { free edge: } m(0)=m^{\prime}(0)=0 \\
\text { simply supported edge: } m(0)=0 \\
\text { clamped edge: no condition }
\end{gathered}
$$

As it is quite apparent from the previous definition, $f^{+}$may be written as

$$
f^{+}=f^{+}(k)
$$

that is, as a function of the sole parameter $k$.

\section{Lower Bound Static Approach}

This approach simply consists of applying the definition of $f^{+}$; that is, in exhibiting particular classes of stress distributions that satisfy both equilibrium and strength requirements. As a first very simple approach, one may consider such distributions where the bending moment is taken to be equal to zero $[m(u)=0]$. Eq. (13) then immediately gives

$$
n(u)=f(1-u)
$$

and the strength condition (14), which reduces here to $|n(u)|$ $\leq 1 u \in[0,1]$, implies that $f \leq 1$.

It follows that

$$
f^{+}(k) \geq 1
$$

for any value of $k \geq 0$.

\section{Upper Bound Kinematic Approach}

This approach results from the dualization of the static approach by means of the virtual work principle, which makes use of kinematically admissible velocity fields for the problem. Any such field that respects the condition of axisymmetry of the problem is characterized in each point of the shell by a set of two kinematic variables. These variables are the component of the velocity along the radial direction, denoted by $v$, and the rate of rotation $\omega$ of the normal to the shell at the same point, counted positive along the orthoradial direction. Owing to the symmetry of the problem, $v$ and $\omega$ are functions of the $z$-coordinate only (Fig. 4). Note that these two variables are a priori independent.

The principle of virtual work states that, given any statically admissible stress distribution of $N_{\theta \theta}, M_{z z}$, and $V_{z}$ in the shell, the following equality holds:

$$
W_{e}(v, \omega)+W_{i}(v, \omega)=0 \quad \text { whatever }(v, \omega)
$$




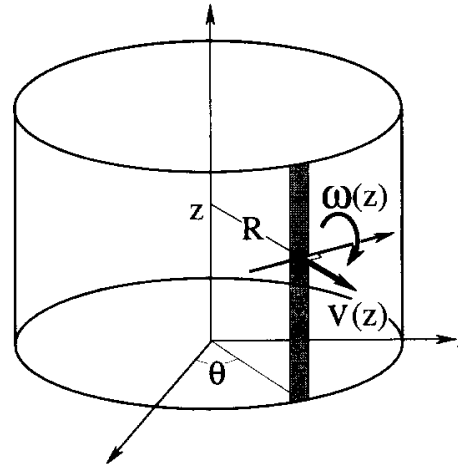

FIG. 4. Virtual Velocity Fields Considered for Problem

where $(v, \omega)$ denotes any kinematically admissible velocity field; and $W_{e}=$ work done by external forces

$$
W_{e}(v, \omega)=2 \pi R \int_{0}^{H} p(z) v(z) d z
$$

while $W_{i}$ represents work corresponding to internal forces (stress distribution), which can be written as

$$
W_{i}(v, \omega)=2 \pi R \int_{0}^{H}-\left[V_{z}\left(\frac{d v}{d z}-\omega\right)+N_{\theta \theta} \frac{v}{R}+M_{z z} \frac{d \omega}{d z}\right] d z
$$

Introducing

$\Pi(v, \omega)=\sup \left\{V_{z}\left(\frac{d v}{d z}-\omega\right)+N_{\theta \theta} \frac{v}{R}+M_{z z} \frac{d \omega}{d z} ;\right.$

with $V_{2}, N_{\theta \theta}$, and $M_{z z}$ satisfying (11) $\}$

it turns out that a necessary condition for the structure to remain stable (i.e., $P \leq P^{+}$) is

$$
W_{e}(v, \omega) \leq W_{m r}(v, \omega) \quad \text { whatever }(v, \omega)
$$

where $W_{m r}(v, \omega)$ represents maximum resisting work defined as

$$
W_{m r}(v, \omega)=2 \pi R \int_{0}^{H} \Pi(v, \omega) d z
$$

with

$$
\begin{aligned}
& \Pi\left(v, \omega=\frac{d v}{d z}\right)=N_{0} \frac{|v|}{R}+M_{0}\left|v^{\prime \prime}\right| \\
& \text { for } \omega=\frac{d v}{d z}=v^{\prime} \text { (Kirchhoff-Love condition) }
\end{aligned}
$$

$\Pi(v, \omega)=+\infty$ otherwise; i.e., when $\omega \neq v^{\prime}$ since the adopted strength condition involves no limitation on the shear component $V_{z}$.

The calculation of $W_{m r}$, and hence the kinematic approach, can be extended to velocity fields exhibiting discontinuities of both $v$ and $\omega$, denoted by $[v]_{i}$ and $[\omega]_{i}$, respectively, along a finite number of points along the vertical direction $(i=1-n)$. It can be shown that the following quantity is to be added to the integral (27):

$$
\sum_{i=1}^{n} \Pi\left([v]_{i},[\omega]_{i}\right)
$$

where

$\Pi\left([v]_{i},[\omega]_{i}\right)=\sup \left\{V_{z}[v]_{i}+M_{z z}[\omega]_{i} ;\right.$ with $V_{z}, M_{z z}$, and $N_{\theta \theta}$ verifying (11)\}
That is

$$
\Pi\left([v]_{i},[\omega]_{i}\right)=+\infty \text { if }[v]_{i} \neq 0
$$

and

$$
\Pi\left([v]_{i},[\omega]_{i}\right)=\Pi\left(\left[v^{\prime}\right]_{i}\right)=M_{0}\left|\left[v^{\prime}\right]_{i}\right| \text { if }[v]_{i}=0
$$

taking into account the fact that the Kirchhoff-Love condition holds on both sides of the discontinuity. This means that the only relevant discontinuities are such that $v$ remains continuous, whereas its first derivative (which coincides with the rate of rotation) may undergo jumps ("hinges") across horizontal circles drawn on the shell surface. Thus

$$
W_{m r}(v)=2 \pi R\left\{\int_{0}^{H}\left(\frac{N_{0}}{R}|v|+M_{0}\left|v^{\prime \prime}\right|\right) d z+\sum_{i=1}^{n} M_{0}\left|\left[v^{\prime}\right]_{i}\right|\right\}
$$

Since $W_{e}(v)=2 \pi R P \int_{0}^{H}(1-z / H) v d z$, one finally gets from (26) with $P=P^{+}$

$$
P^{+} \leq \frac{W_{m r}(v)}{2 \pi R \int_{0}^{H}\left(1-\frac{z}{H}\right) v d z}
$$

provided that $\int_{0}^{H}(1-z / H) v d z$ be strictly positive. The inequality in (33) clearly shows that the kinematic approach provides upper-bound estimates for the ultimate load $P^{+}$.

\section{CASE OF CYLINDER WITH FREE LOWER EDGE}

This case will first be dealt with by means of the kinematic approach, then through the static approach, thus obtaining the exact solution for the problem.

\section{Kinematic Approach}

The class of velocity yields $v$ which we will consider is shown in Fig. 5.

It involves a hinge circle separating the upper part of the shell that remains motionless from the lower part, which is given a motion defined by

$$
v(z)=\alpha\left(1-\frac{z}{z_{1}}\right) ; \quad 0 \leq z \leq z_{1} \leq H
$$

$(34 a, b)$

where $\alpha=$ positive constant. The value of the discontinuity of $v^{\prime}$ is therefore

$$
\left[v^{\prime}\right]\left(z_{1}\right)=v^{\prime}\left(z_{1}^{+}\right)-v^{\prime}\left(z_{1}^{-}\right)=\alpha / z_{1}
$$

Hence, the maximum resisting work is given by (32) where $W_{m r}(v)=2 \pi R\left[M_{0}\left(\alpha / z_{1}\right)+\alpha\left(N_{0} / R\right)\left(z_{1} / 2\right)\right]$ and then from (34), simplifying by $\alpha, P^{+} \leq\left(M_{0} / z_{1}+N_{0} z_{1} / 2 R\right) /\left(z_{1} / 2\right)\left(1-z_{1} / 3 H\right)$ or, put in nondimensional form

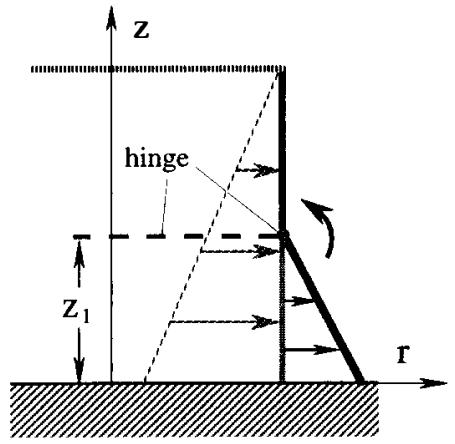

FIG. 5. Failure Mechanism with Circular Hinge 


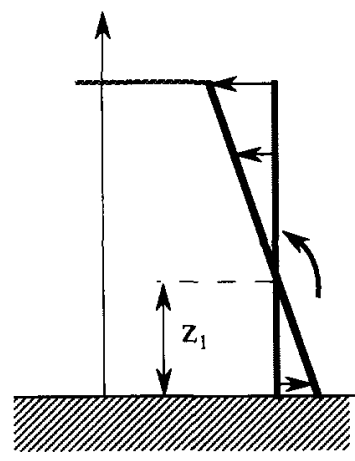

FIG. 6. Fallure Mechanism without Circular Hinge

$f^{+} \leq \frac{1+2 k / u_{1}^{2}}{1-u_{1} / 3}=f_{1}^{\mu}\left(u_{1}, k\right) \quad$ with $u_{1}=z_{1} / H \quad u_{1} \in[0,1]$

The minimal value of the right member, that is, the optimal upper-bound value of $f^{+}$derived from this particular class of mechanisms, is obtained for $u_{1}=u_{1}^{*}$ such that

$$
k=\frac{u_{1}^{*^{3}}}{6\left(2-u_{1}^{*}\right)}
$$

hence

$$
f^{+} \leq f_{1}^{\mu}\left[u_{1}^{*}(k), k\right]=f_{1}^{u}(k) \quad \text { with } f_{1}^{u}(k)=\frac{2}{2-u_{1}^{*}}
$$

Consequently, the optimal upper bound is given in the following implicit form as the solution of:

$$
k=\frac{2}{3} \frac{\left(f_{1}^{\mu}-1\right)^{3}}{\left(f_{1}^{u}\right)^{2}}
$$

This approach is completed by considering another class of mechanisms where no circular hinge is involved, as shown in Fig. 6.

The motion is defined by

$$
v(z)=\alpha\left(1-\frac{z}{z_{1}}\right) \text { for } 0 \leq z \leq H
$$

Performing the same calculations as before, one obtains

$$
W_{m r}(v)=2 \pi \alpha N_{0} H\left[u_{1}-\left(1-\frac{1}{2 u_{1}}\right)\right] \text { if } u_{1} \leq 1
$$

We get the following upper bound:

$$
f^{+} \leq 3 \frac{\left(2 u_{1}^{2}-2 u_{1}+1\right)}{\left(3 u_{1}-1\right)}=f_{2}^{u}\left(u_{1}, k\right)
$$

which is valid for $1 / 3 \leq u_{1} \leq 1$, and whose minimum value, obtained for $u_{1}^{*}=[(\sqrt{10}+2) / 6]$, is

$$
f^{+} \leq f_{2}^{u}=\frac{2(\sqrt{10}-1)}{3} \approx 1.4415
$$

Fig. 7 represents the curves giving the variations of both upper bounds $f_{1}^{u}$ and $f_{2}^{u}$ as functions of parameter $k$. It follows that the best upper-bound estimate that can be derived from the two previous kinds of mechanisms is

$f^{+} \leq f^{u}(k)=\min \left\{f_{1}^{u}, f_{2}^{u}\right\} \quad$ with $f^{u}(k)=f_{1}^{u}(k)$ solution of (39)

if $k \leq 0.0276$ and $f_{2}^{u} \approx 1.4415$ otherwise

$(44 a-c)$

\section{Static Approach}

It will now be demonstrated that $f^{+}=f^{u}$ by finding out the corresponding distributions of normal force $N_{\theta \theta}$ and bending moment $M_{z z}$, which are in equilibrium with $f^{u}$, while comply-

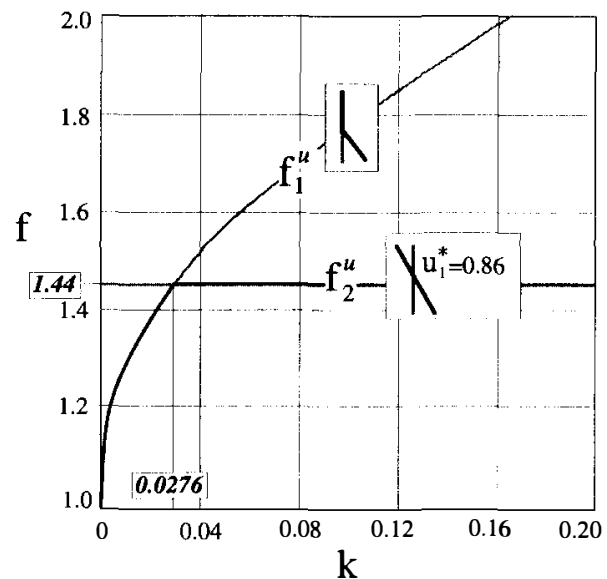

FIG. 7. Plotting of $f_{1}^{u}$ and $f_{2}^{u}$ as Functions of $k$

ing with the strength criterion everywhere. This can be achieved by using the theorem of association (Salençon 1983) as a guideline. More specifically, in the present case, this theorem states that if any such stress distribution exists, it necessarily satisfies $N_{\theta \theta}=+N_{0}$ (or $n=+1$ ) in the lower part of the structure, since the associated strain rate component $v / R$ in the optimal mechanism is positive. Therefore, the equilibrium (13) combined with the boundary conditions (16) gives

$$
m\left(u<u_{1}^{*}\right)=m^{-}(u)=\frac{1}{k}\left[\left(f^{u}-1\right) \frac{u^{2}}{2}-f^{u} \frac{u^{3}}{6}\right]
$$

It can be easily seen that this distribution is compatible with the strength condition $\left(\left|\mathrm{m}^{-}\right| \leq 1\right)$. It may be completed in the upper part $\left(u \geq u_{1}^{*}\right)$ by two kinds of distributions.

\section{Sinusoidal Distribution}

We consider the distribution defined by

$$
m^{+}(u)=\frac{1}{2}\left[\cos \left(\pi \frac{u-u_{1}^{*}}{1-u_{1}^{*}}\right)+1\right] \quad u \geq u_{1}^{*}
$$

This distribution verifies $\left|m^{+}\right|<1$, as well as the continuity of $m$ and $m^{\prime}$ at the connecting point $u_{1}^{*}$ with $m^{-}$and the boundary conditions on the upper edge $\left[m(1)=m^{\prime}(1)=0\right]$. Then, the equilibrium (13) makes it possible to calculate the corresponding distribution of $n$, denoted by $n^{+}$

$$
n^{+}(u)=f^{u}(1-u)+\frac{k}{2} \frac{\pi^{2}}{\left(1-u_{1}^{*}\right)^{2}} \cos \left(\pi \frac{u-u_{1}^{*}}{1-u_{1}^{*}}\right)
$$

A sufficient condition for this distribution to obey the strength condition $\left(\left|n^{+}\right| \leq 1, u_{1}^{*} \leq u \leq 1\right)$ is

$$
k \leq \frac{2}{\pi^{2}} \frac{\left(f^{u}-1\right)\left(2-f^{u}\right)^{2}}{\left(f^{u}\right)^{2}}
$$

that is, taking into account the relationship between $f^{u}$ and $k$

$$
k \leq 0.01629
$$

It follows that

$$
f^{+}(k)=f^{u}(k) \text { for } k \leq 0.01629
$$

\section{Polynomial Distribution}

This kind of distribution is obtained by choosing $n^{+}=a=$ $c t$, so that integrating the equilibrium (13) and using the boundary conditions for $u=1$

$$
m^{+}=\frac{1}{k}\left[\frac{f_{1}^{u}}{6}(1-u)^{3}-\frac{a}{2}(1-u)^{2}\right]
$$



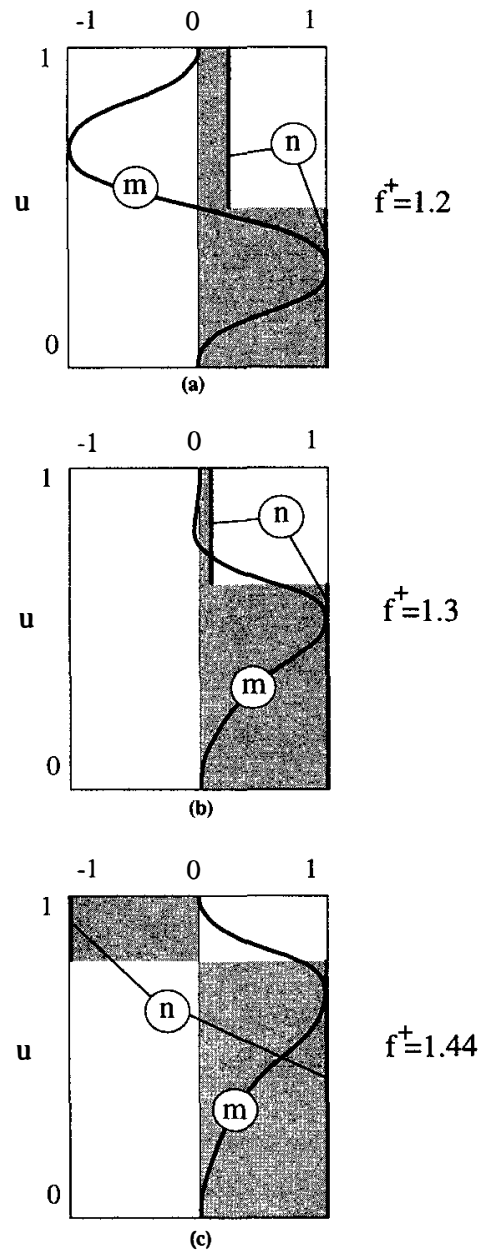

FIG. 8. Three Examples of Distributions of $m(u)$ and $n(u)$ in Equilibrium with: (a) $f^{+}(k)=1.2$, (b) $f^{+}(k)=1.3$, and $(c) f^{+}(k)=$ 1.4415

The value of parameter $a$ may be determined by enforcing the continuity of $m$ and $m^{\prime}$ at the connecting point between $m^{+}$and $m^{-}$

$$
a=f^{u}\left(\frac{3 f^{u}-4}{8 f^{u}-12}\right)
$$

This distribution respects the strength condition $(|a| \leq 1$ and $\left.\left|m^{+}\right| \leq 1\right)$ if and only if

$$
1.2 \leq f^{u}(k) \leq 1.4415
$$

or

$$
0.0037 \leq k \leq 0.0276
$$

and then

$$
f^{+}(k)=f^{u}(k) \quad \text { if } 0.0037 \leq k \leq 0.0276
$$

Finally, since it can be easily seen that $f^{+}$increases with $k$, (50) and (55) lead to

$$
f^{+}=f^{u}(k) \text { given by (44) for } k \geq 0
$$

For illustrative purposes, the corresponding distribution of $n(u)$ and $m(u)$ has been drawn in Fig. 8 for several values of $f^{+}(k)$.

\section{CYLINDER WITH SIMPLY SUPPORTED OR CLAMPED EDGE}

The equations governing the problem are the same as those in the preceding case, except that the boundary conditions applying at the bottom edge are now

$$
m(0)=0 ; \quad v(0)=0
$$

for the case of the simply supported edge and

$$
v(0)=v^{\prime}(0)=0
$$

in the case of the clamped edge.

The same kind of approach as that previously developed for a free bottom edge is followed. The failure mechanisms explored in the kinematic approach are represented in Fig. 9. They involve up to three hinge circles, with the lowest one, located at the bottom of the cylinder, implying no contribution to the maximum resisting work for a simply supported edge. They produce upper-bound estimates for $f^{+}(k)$, which can be proved to be the exact solution by exhibiting associated stress distributions given in Appendices I and II. As an example, such a distribution, along with the associated failure mechanism, is shown in Fig. 10 for the case of a simply supported cylinder.

The results of the present study are gathered in Fig. 11, in the form of curves giving the exact value of $f^{+}$as a function of $k$ for the three different boundary conditions.

As could be anticipated, the greatest value of $f^{+}(k)$ is obtained for the case of a clamped edge, the intermediate curve corresponding to the simply supported edge which remains free to rotate about the orthoradial axis.

\section{BACK TO INITIAL PROBLEM}

Resuming the analysis of the cellular cofferdam undertaken in an earlier section of the present paper, it appears that the stability of the composite structure (backfill material + sheet pile wall) under its own weight is ensured provided that

$$
\gamma H R / N_{0} \leq\left(\gamma H R / N_{0}\right)^{+}
$$

where $\left(\gamma H R / N_{0}\right)^{+}$denotes the ultimate value of $\gamma H R / N_{0}$ beyond which failure will occur. It may be easily seen from dimensional analysis arguments that

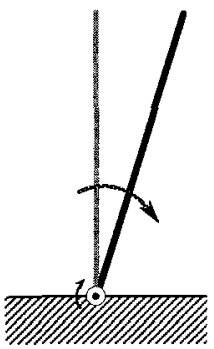

(a)

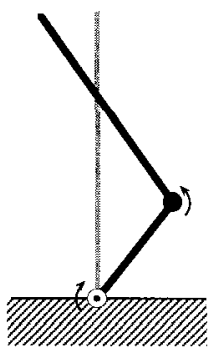

(b)

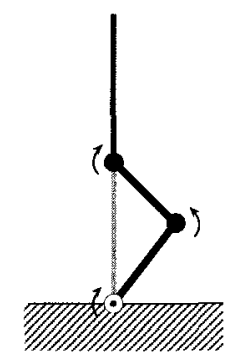

FIG. 9. Failure Mechanisms Considered in Case of Simply Supported or Clamped Bottom Edge: (a) One Hinge Circle; (b) Two Hinge Circles; (c) Three Hinge Circles

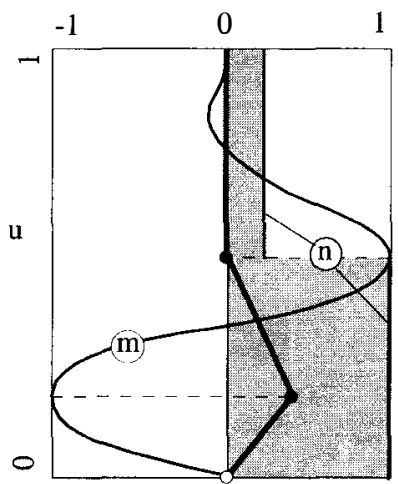

FIG. 10. Stress Distribution and Associated Mechanism for Simply Supported Cylinder $\left[f^{+}(k)=1.6\right]$ 


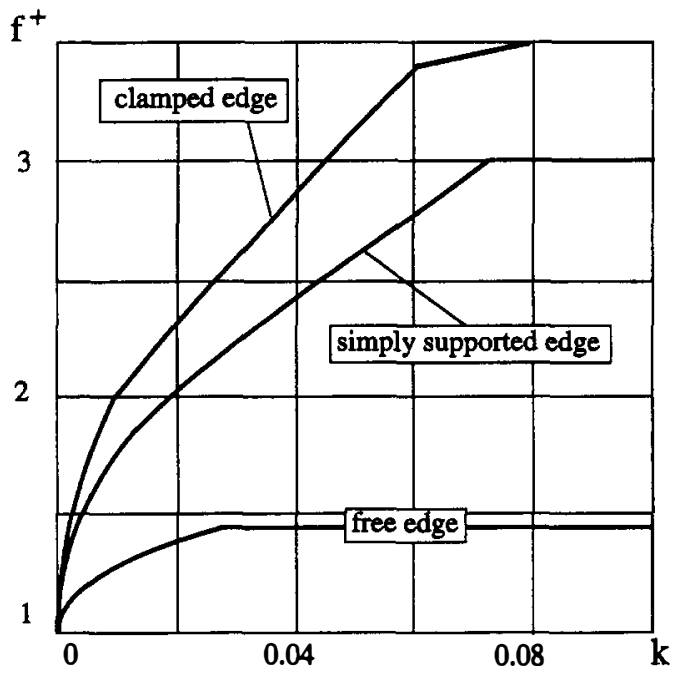

FIG. 11. Ultimate Load $f^{+}$versus Parameter $k$ for Different Boundary Conditions at Bottom of Shell

$$
\left(\gamma H R / N_{0}\right)^{+}=F^{+}\left(\varphi, \frac{H}{R}, \frac{M_{0}}{N_{0} R}\right)
$$

Referring again to the lower-bound static approach, where stress fields of the form (7) are considered in the backfill material, it can be deduced from the previous analysis of the auxiliary problem that a sufficient condition for the sheet pile wall to resist to the linear pressure distribution (10) associated with those stress fields, writes

$$
P=\lambda y H \leq P^{+}=\frac{N_{0}}{R} f^{+}\left(k=\frac{M_{0} R}{N_{0} H^{2}}\right)
$$

that is

$$
\frac{\gamma H R}{N_{0}} \leq \frac{f^{+}(k)}{\lambda}
$$

Thus, given any value of $\lambda$ ranging between $K_{a}$ and $K_{p}$ [see (8)], so as to ensure that the stress field in the backfill material complies with strength condition $(1) f^{+}(k) / \lambda$ clearly provides a lower bound estimate for $\left(\gamma H R / N_{0}\right)^{+}$. The best (i.e., the greatest) estimate is obviously obtained by choosing the minimum value of $\lambda$, which is $K_{a}$. Hence

$$
\left(\frac{\gamma H R}{N_{0}}\right)^{+} \geq \frac{f^{+}}{K_{a}}=K_{p} f^{+}
$$

This represents a significant improvement of the result already obtained by Dormieux and Delaurens (1991) by taking $f^{+}=1$; that is, by implicitly neglecting the resistance of the confining shell to bending moments $(k=0)$.

A numerical example will help to assess this improvement. A cellular cofferdam of height $H=20 \mathrm{~m}$ and radius $R=7.5$ $\mathrm{m}$ is built up by using sheet piles having a thickness of $e=$ $0.012 \mathrm{~m}$ made of a steel, the yield strength of which is equal to $\sigma_{0}=250 \mathrm{MPa}$ so that

$$
\begin{gathered}
N_{0}=\sigma_{0} e=3 \mathrm{MN} / \mathrm{m} ; \quad M_{0}=\sigma_{0} e^{2} / 4=9 \mathrm{kNm} / \mathrm{m} \\
\text { and } k=\frac{M_{0} R}{N_{0} H^{2}} \approx 5.6210^{-5}
\end{gathered}
$$

The corresponding values of $f^{+}(k)$ reported in Fig. 11 are

- 1.045 for a free bottom edge

- 1.102 for a simply supported edge

- 1.119 for a clamped edge

That is an improvement of up to $12 \%$ with respect to the simplified lower-bound estimate of Dormieux and Delaurens.

\section{CONCLUDING REMARKS}

By adopting a simplified expression for the yield criterion of an axisymmetrically loaded shell expressed in terms of normal forces and bending moments, exact solutions to the problem of a cylindrical shell submitted to a linearly varying internal pressure have been developed. In particular, it has been shown that the ultimate load increases with a parameter $k$, which characterizes the relative thickness of the shell. Moreover, the importance of the boundary conditions prescribed at the lower edge of the shell has been clearly pointed out. As an example, the improvement of the lower-bound estimate for the stability factor of a single cellular cofferdam resulting from such an analysis may range between $5 \%$ and $12 \%$ when compared to previous conservative estimates where the resistance to bending moments is neglected. It should be emphasized that such improved lower-bound estimates remain valid for any strength condition governing the interface between the sheet pile wall and the backfill material. In other terms, taking into account nonzero shear stresses $T$ along this interface and, hence, more complex stress fields in the backfill zone than those considered in the prior analysis (namely with $\sigma_{z \theta} \neq 0$ ) would necessarily result in deriving still better lower bounds. Likewise, upper-bound estimates for the stability factor could be expected from the kinematic approach. This would necessitate constructing relevant velocity fields both in the backfill material and in the surrounding shell.

\section{APPENDIX I. RESULTS FOR CYLINDER WITH SUPPORTED EDGE}

$$
\begin{aligned}
& \text { If } k \leq 0.01276 \text {, we have the following results: } \\
& \qquad \begin{array}{l}
k=\frac{\sqrt{3}}{27} \frac{(f-1)^{3}}{f^{2}} ; \quad u_{1}=\frac{3-\sqrt{3}}{3} \frac{f-1}{f} \quad(65 a, b) \\
u_{2}=\frac{3+\sqrt{3}}{3} \frac{f-1}{f} \text { and } \\
m_{b}=-3 \sqrt{3} \frac{f}{f-1} u+9 \sqrt{3} \frac{f^{2}}{(f-1)^{3}}\left[(f-1) \frac{u^{2}}{2}-f \frac{u^{3}}{6}\right]
\end{array}
\end{aligned}
$$

where $u_{1}$ and $u_{2}$ denote loci of the two hinges. The distribution $m_{b}$ must be completed in the upper part by two kinds of distributions

$$
\begin{gathered}
m_{s}=\frac{1}{2}\left[\cos \left(\pi \frac{u-u_{2}}{1-u_{2}}\right)+1\right] \\
m_{p}=\frac{1}{k}\left[(f-a) \frac{u^{2}}{2}-f \frac{u^{3}}{6}+\frac{1}{2}(2 a-f) u-\frac{1}{6}(3 a-f)\right] \\
\text { with } a=-\frac{f^{4}-4 f^{3}+12 f^{2}-16 f+4}{12 f(2-f)}
\end{gathered}
$$

It can be proved that the distribution $m_{s}$ gives a static solution for $1 \leq f \leq 1.759(0 \leq k \leq 0.009061)$ and that $m_{p}$ gives a static solution for $1.421 \leq f \leq 1.893(0.002371 \leq k \leq$ 0.01276).

If $0.01276 \leq k \leq 0.07407$, we have the following results with $m_{1}$, the moment distribution, in the lower part $\left(u \leq u_{r}\right)$ and $m_{2}$ for the upper part

$$
\begin{aligned}
k= & \frac{1}{18 f^{2}} \\
& \cdot\left\{\begin{array}{l}
\left(6-36 f+27 f^{2}+3 f^{3}-6 \sqrt{6} \sqrt{f+3} f^{2}+6 \sqrt{6} \sqrt{f+3} f\right) \\
+\sqrt[4]{6}\left[4(f+3)^{3 / 2}-4 \sqrt{6}(f+3)-12 \sqrt{f+3}+13 \sqrt{6}\right]^{3 / 2}
\end{array}\right\}
\end{aligned}
$$




$$
\begin{aligned}
& m_{1}=\frac{1}{k}\left[(f-1) \frac{u^{2}}{2}-f \frac{u^{3}}{6}+\left(\sqrt{\frac{2}{3}} \sqrt{f+3}-\frac{f+2}{2}\right) u\right] \\
& m_{2}=\frac{1}{k}\left[(f+1) \frac{u^{2}}{2}-f \frac{u^{3}}{6}-\frac{1}{2}(f+2) u+\frac{1}{2}(f+3)\right] \\
& u_{r}=\sqrt{\frac{f+3}{6}} ; \quad u_{1}=\frac{f-1}{f}-\sqrt{\left(\frac{f-1}{f}\right)^{2}+\sqrt{\frac{8}{3}} \frac{\sqrt{f+3}}{f}-\frac{2}{f}-1}
\end{aligned}
$$

If $k \geq 0.7407$, we have the following results:

$$
f=3 ; \quad m=\frac{1}{k}\left(-\frac{u^{3}}{2}+u^{2}-\frac{u}{2}\right)
$$

\section{APPENDIX II. RESULTS FOR CYLINDER WITH CLAMPED EDGE}

If $k \leq 0.01108$, we have the following results:

$$
\begin{aligned}
& k=\frac{1}{24} \frac{(f-1)^{3}}{f^{2}} ; \quad u_{1}=\frac{f-1}{2 f} ; \quad u_{2}=\frac{3}{2} \frac{f-1}{f} \text { and } \\
& m_{b}=1-9 \frac{f-1}{f} u+24 \frac{f^{2}}{(f-1)^{3}}\left[(f-1) \frac{u^{2}}{2}-f \frac{u^{3}}{6}\right]
\end{aligned}
$$

The distribution $m_{b}$ must be completed in the upper part of the shell by two kinds of distributions

$$
\begin{gathered}
m_{s}=\frac{1}{2}\left[\cos \left(\pi \frac{u-u_{2}}{1-u_{2}}\right)+1\right] \\
m_{p}=\frac{1}{k}\left[(f-a) \frac{u^{2}}{2}-f \frac{u^{3}}{6}-\frac{1}{2}(f-2 a) u+\frac{1}{6}(f-3 a)\right] \\
\text { with } a=\frac{3 f^{4}-12 f^{3}+42 f^{2}-60 f+11}{16\left(3 f^{2}-6 f-1\right)}
\end{gathered}
$$

It can be proved that $m_{s}$ gives a static solution for $1 \leq f \leq$ $1.8762(0 \leq k \leq 0.00796)$ and that $m_{p}$ gives a static solution for $1.486 \leq f \leq 2.0315$ (0.002167 $\leq k \leq 0.01108)$.

If $0.01108 \leq k \leq 0.05927$, it can be proved that $k$ is the only positive root of the following equation:

$$
\begin{aligned}
& k^{4}\left(559872 f^{4}\right)+k^{3}\left(-186624 f^{5}-559872 f^{4}-1990656 f^{3}\right. \\
& \left.+27648 f^{2}\right)+k^{2}\left(23328 f^{6}+46656 f^{5}+728352 f^{4}\right. \\
& \left.+432000 f^{3}+2125440 f^{2}-82944 f\right) \\
& +k\left(-1296 f^{7}+3888 f^{6}-5616 f^{5}-108720 f^{4}\right. \\
& \left.+27648 f^{3}+680832 f^{2}-601344 f+62208\right) \\
& +\left(27 f^{8}-324 f^{7}+450 f^{6}+4372 f^{5}-11565 f^{4}\right. \\
& \left.-9432 f^{3}+49464 f^{2}-38880 f+3888\right)=0
\end{aligned}
$$

This equation can be algebraically solved, but it is more convenient to use numerical methods. We denoted by $m_{1}$ the moment distribution in the lower part $\left(u<u_{r}\right)$, and by $m_{2}$ the moment distribution in the upper part. The expressions of $m_{1}$ and $m_{2}$ are given next

$$
\begin{aligned}
m_{1} & =1+\frac{1}{k}\left[(f-1) \frac{u^{2}}{2}-f \frac{u^{3}}{6}\right. \\
& \left.+\frac{-(f+2)+\sqrt{\frac{8}{3}(f+3)-16 k}}{2} u\right]
\end{aligned}
$$

$$
\begin{gathered}
m_{2}=\frac{1}{k}\left[(f+1) \frac{u^{2}}{2}-f \frac{u^{3}}{6}-\frac{1}{2}(f+2) u+\frac{f+3}{6}\right] \text { and } \\
u_{r}=\frac{1}{4} \sqrt{\left.\frac{8}{3}(f+3)-16 k\right)}
\end{gathered}
$$

If $k \geq 0.059217$, the study has been completely carried out by Olszak and Sawczuk (1959). The results are

$$
k=\frac{f-3}{6} ; \quad m=1+\frac{1}{k}\left[(f-1) \frac{u^{2}}{2}-f \frac{u^{3}}{6}-\frac{(f-2)}{2}\right]
$$

\section{APPENDIX III. REFERENCES}

Bowles, J. E. (1977). Foundation analysis and design. McGraw-Hill Book Co., Inc., New York, N.Y.

Chen, W. F., and Han, D. J. (1988). Plasticity for structural engineers. Springer-Verlag New York, Inc., New York, N.Y.

Dormieux, L., and Delaurens, C. (1991). "Stabilité d'une cellule de gabion sous poids propre." Revue Française de Géotechnique, 55, 4761 (in French).

Drucker, D. C. (1954). "Limit analysis of cylindrical shells under axiallysymmetric loading." Proc., Ist Midw. Conf. Solid. Mech., ASME, New York, N.Y., 158-163.

Drucker, D. C., and Shield, R. T. (1959). "Limit analysis of symmetrically loaded thin shells of revolution." J. Appl. Mech., 26(March), 61-68.

Eason, G., and Shield, R. T. (1955). "The influence of free ends on the load carrying capacities of cylindrical shells." J. Mech. and Phys. of Solids, 4, 17-27.

Hodge, P. G. Jr. (1954). "The rigid-plastic analysis of symmetrically loaded shells." J. Appl. Mech., 21, 336-342.

Hodge, P. G. Jr. (1963). Limit analysis of rotationnally symmetric plates and shells. Prentice-Hall ser. in Theoretical and Appl. Mech., PrenticeHall Inc., Englewood Cliffs, N.J.

Lacroix, Y., Esriq, M. I., and Luscher, U. (1970). "'Design, construction, and performance of cellular cofferdams." Proc., Lateral Stresses in the Ground and Design of Earth-Retaining Structures, ASCE, New York, N.Y., $271-328$.

Olszak, W., and Sawczuk, A. (1959). Die grenztragfähigkeit von zylindrischen schalen bei verscheidenen formen der plastizitätsbedingung." Acta Technica Academice Scientiarum Hungaricae, t XXVI, (1-2), 55-77 (in German).

Onat, E. T., and Prager, W. (1954). "Limit analysis of shells of revolution." Proc., Roy. Netherlands Acad. Sci., series B, 57, 534-548.

Salençon, J. (1983). Calcul á la rupture et analyse limite. Presses de l'École Nationale des Ponts et Chaussées, Paris, France (in French).

Salençon, J. (1990). "An introduction to the yield design theory and its application to soil mechanics." Eur: J. Mech., A/Solids, 5(9), 477-500.

Salençon, J. (1993). "'Yield design: a survey of the theory."' Evaluation of global bearing capacity of structures. Springer-Verlag New York, Inc., New York, N.Y.

Save, M., and Massonnet, C. (1972). Calcul plastique des constructions. Vol. II, Structures dépendant de plusieurs paramétres, CBLIA, Bruxelles, Belgium (in French).

\section{APPENDIX IV. NOTATION}

The following symbols are used in this paper:

$e=$ thickness of shell;

$f=$ nondimensional loading parameter $\left(P R / N_{0}\right)$;

$f^{+}=$ultimate value of $\left(P R / N_{0}\right)$;

$H=$ height of cylinder;

$k=$ nondimensional characteristic parameter of cylindrical shell $\left(M_{0} R / N_{0} H^{2}\right)$;

$K_{a}=$ active earth pressure coefficient;

$K_{p}=$ passive earth pressure coefficient;

$M=$ bending moment;

$M_{0}=$ yield bending moment;

$m=$ reduced bending moment $\left(M / M_{0}\right)$;

$N=$ normal force per unit length;

$N_{0}=$ yield normal force per unit length; 
$n=$ reduced normal force per unit length $\left(N / N_{0}\right) ;$

$p=$ pressure;

$P=$ loading parameter (greatest value of pressure);

$P^{+}=$ultimate value of loading parameter;

$R=$ radius of cylinder;

$\boldsymbol{u}=$ reduced vertical coordinate $(z / H)$;

$v=$ horizontal velocity;

$V=$ shear per unit length;
$W_{e}=$ work of external forces;

$W_{i}=$ work of internal forces;

$W_{m r}=$ maximum resisting work;

$\gamma=$ specific weight of backfill material;

$\Pi=$ density of maximum resisting work;

$\varphi=$ friction angle;

$\sigma_{0}=$ resistance of material under uniaxial solicitation; and

$\omega=$ rate of rotation. 